\title{
Constraints on the asymmetric equation of state from heavy-ion collisions
}

\author{
Sherry Yennello, Alan McIntosh and Lauren Heilborn
}

Department of Chemistry \& Cyclotron Institute, Texas A\&M University, College Station, TX, USA

\begin{abstract}
Nuclear matter is one of the most fascinating materials that exists. Therefore elucidating the equation-of-state of nuclear matter is a fundamentally interesting question. Additionally, the nuclear equationof-state has impacts on astrophysical observables. One important means of constraining the nuclear equation-of-state is through studying heavy-ion collisions. Nuclear material has two components - neutrons and protons - the ratio of which impacts the equation-of-state. Measurements of fragments emitted from reactions of nuclei with different ratios of neutrons and protons - and comparison to simulations based on various underlying interactions - have placed constraints on both the symmetric and asymmetric parts of the equation of state.
\end{abstract}

\section{Introduction}

The equation-of-state for nuclear matter (nEOS) is important for many astrophysical processes and vital to answer the question of how the elements are formed. At the heart of the question is what is the isospin dependent nucleon-nucleon interaction, and how does it get modified when it is in the presence of other nucleons. This interaction affects the neutron star massradius relationship and the cooling rate of neutron stars. $[1,2]$ However the underlying interaction is not directly input to the astrophysical calculations, but rather the nEOS - which is derived from the comparison of observables 
to models with various interactions - is the input to the astrophysical calculations. Recent measurements of 2 solar mass neutron stars have challenged many of the previously accepted nEOS. [3] Additionally, the recent conjecture that the radius of neutron stars around $9 \mathrm{~km}$ requires a much denser core of the neutron star and opens the discussion of deconfined nuclear matter or strangeness that could soften the nEOS at these high densities.

Nuclear matter is one of the most fascinating materials that exists. The constituent nucleons are fermions with a size on the order of a femtometer. The nucleon-nucleon interaction is attractive, but has a hard core repulsion resulting in an energy minimum at a specific distance leading to a preferred, or saturation, density. As the system is moved away from the saturation density an energy cost must be paid. This energy cost can be quantified as the incompressibility of nuclear matter. The strong interaction manifests itself in nuclei in terms of the neutron skin, the ground state masses and the saturation density. Additionally it controls nuclear reaction mechanisms such as multifragmentation, transverse collective flow and transport leading to equilibration. Nuclear matter has often been described as a quantum liquid. At high temperatures there is a transition from a liquid to a gas. At very high temperatures there is a further transition to a deconfined quark gluon plasma. At very low densities clustering occurs. Over a range of densities various interesting shapes of so called nuclear pasta are preferred. These various behaviors are a result of the nEOS, therefore elucidating that nEOS is a fundamentally interesting question. Yet whether we are describing the neutron skin thickness on a single nucleus or the density in a neutron star - which vary in size by many orders of magnitude - one needs a common nEOS. An important mechanism for constraining the nEOS is through studying femtonovae in the lab.

Giant resonance measurements have given us the best constraints on the nEOS for "normal" nuclear material, ie nuclei that are near the valley of stability, relatively cold and not far from saturation density. The incompressibility of nuclear matter, $\mathrm{K}_{N M}$, is related to the excitation of the GMR of finite nuclei, but one must account for surface, Coulomb and asymmetry contributions. Measurements taken over a variety of stable nuclei have constrained the incompressibility near the valley of stability. In 1999, measurements of the ISGMRs [4] were compared to HF-based RPA calculations which used the Gogny interaction and took into account pairing and anharmonicity corrections, and a value of $\mathrm{K}_{N M} \sim 230 \mathrm{MeV}$ was obtained. The measurement of the GMR for nuclei with increasing asymmetry can help refine the $\mathrm{K}_{N M}$. [5] Moving away from saturation density, the data from the measurement of transverse collective flow has been used to constrain 


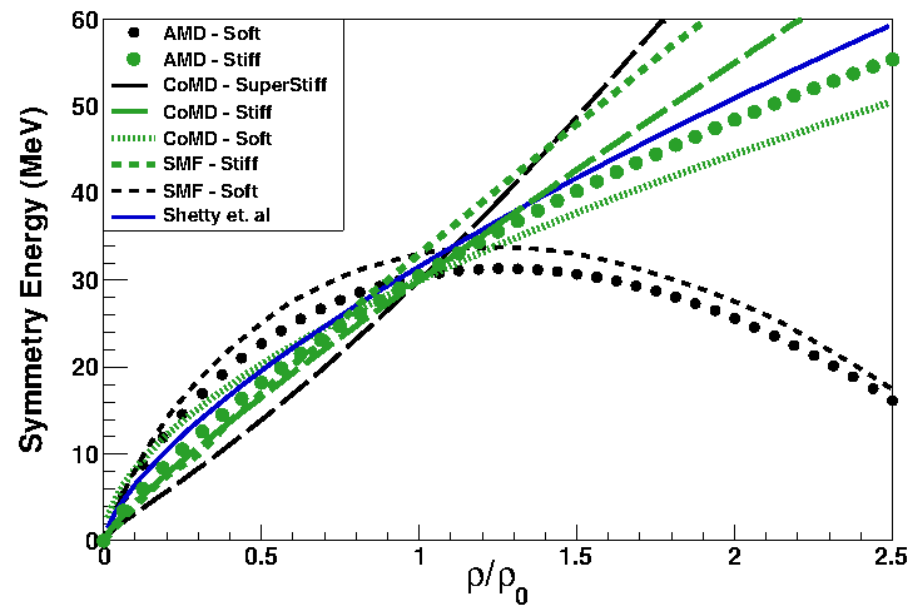

Figure 1: Various forms of the density dependence of the symmetry energy as implemented in the AMD, CoMD and SMF models. Additionally shown is the experimental data from Shetty. [7]

the nEOS at up to $\rho / \rho_{0}=1.5$. [6] However this data is unable to address what happens away from the valley of stability. As one moves away from the valley of stability to more neutron-rich systems the symmetry energy must be taken into account in the nEOS. The magnitude and shape of the symmetry energy depends on the underlying interaction. Many different curves result from different model calculation as shown in figure 1. These are often broadly classified into "soft", where the symmetry energy versus density curve is convex and turns over at high density, and "stiff", where the symmetry energy continues to increase as a function of density. Since these are relative terms it is more accurate to specify value, slope and curvature at saturation density. While the symmetry energy is often characterized by the behavior near saturation density it must be recognized that there could be a softening at higher density or a flattening of this curve at lower density. This paper will discuss the progress that has been made on constraining the asymmetric part of the nEOS away from saturation density using heavy-ion collisions.

\section{Constraints on nEOS from isoscaling}

The nEOS directly impacts the fragmentation of hot nuclei. When examining the breakup of two equilibrated nuclear systems that are nearly iden- 
tical in all aspects except for the neutron-to-proton ratio one can extract a measure of the nuclear symmetry energy through isoscaling. The work of Tsang [8] demonstrated that scaling appears when you compare the ratios of fragment yields between a neutron-rich and a neutron-poor system. Ono used this technique to analyze the fragments produced from AMD calculations for two underlying interactions and proposed the relationship as the first observable that could constrain the nEOS. [9] This calculation was compared with experimental data to put forth the first constraints on the symmetry energy of the nEOS from heavy-ion collisions. [10]

Such indirect measurements depend on comparison to theoretical calculations in order to extract the symmetry energy. This technique was used with experimental data as well as statistical calculations and dynamical calculations to map how the symmetry energy changes as a function of excitation energy. A decrease in symmetry energy as a function of excitation energy has been linked with a decrease in the density. [11] In order to extract a meaningful value of symmetry energy from isoscaling one must accurately know the $(N-Z) / A$ of the sources and the temperature of the system. Recent work by McIntosh [12-15] has demonstrated that there is an $(N-Z) / A$ dependence in the nuclear caloric curve. One also must take into account any secondary cooling or experimental filters that are needed to map the observed fragments to primary fragments. In order to better define the source Wuenschel [16] and Galanopolous [17] developed the technique of source reconstruction of the fragmenting quasiprojectile. Another technique for connecting the fragment yield ratios to the symmetry energy is through the Landau expansion of the free energy near the critical point using mirror nuclei. This work extracts a symmetry energy versus excitation energy that is consistent with that extracted from isoscaling. [18, 19] Isoscaling of data from many experiments and theoretical simulations has provided initial constraints on the symmetry energy of the nEOS. These constraints have been augmented by measurements of other observables, such as equilibration and fragment flow.

\section{Nucleon transport as measured by isospin equilibration}

The amount of mixing of nucleons between two reacting systems is a result of the underlying nucleon transport, which is dependent on the symmetry energy of the nEOS. Early measurements using isotopically identified fragments from collisions of isobaric nuclei were used to measure the onset of 
isospin non-equilibration. [20,21] At higher energies this non-equilibration was quantified through development of the isospin tracer method. [22] The underlying nucleon transport was broken down into drift and diffusion by Shi [23] and the diffusion coefficient was connected to the symmetry potential. Tsang used the tracer method to measure isospin diffusion and through comparison to theoretical calculations determined the slope of the symmetry energy near saturation density to be $\mathrm{L}=88 \pm 25 \mathrm{MeV}$.
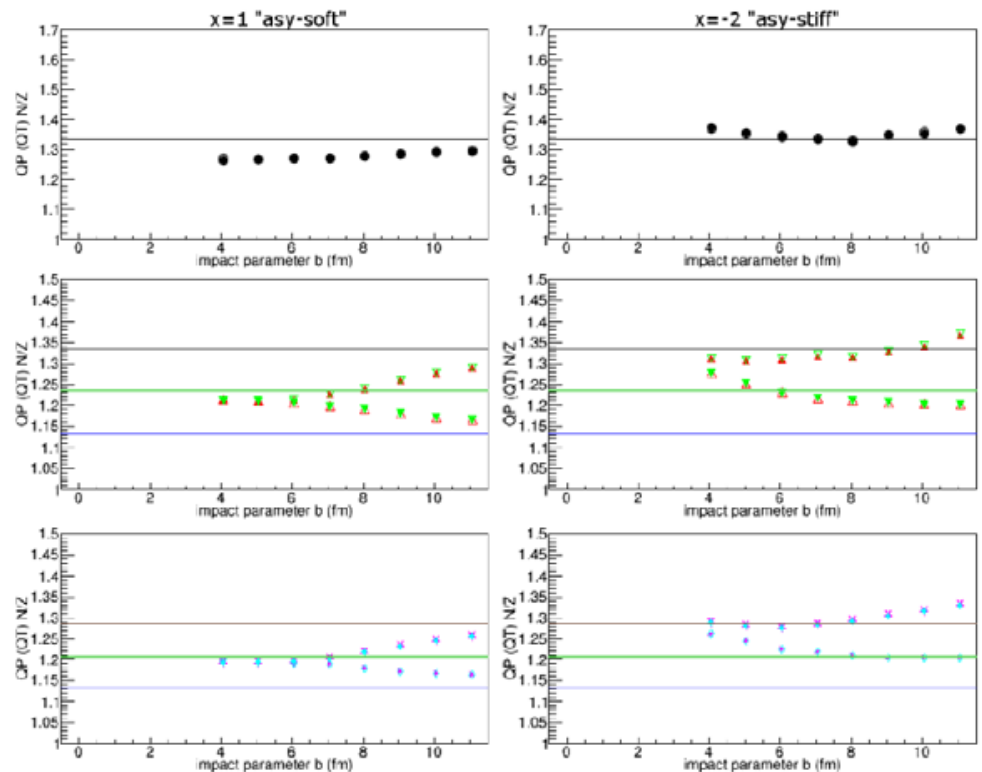

Figure 2: QP (solid symbol) and QT (open symbol) N/Z values as a function of impact parameter. The left and right side correspond to the "asy-soft" and "asy-stiff" parameterizations of the symmetry energy, respectively. Top row: ${ }^{70} \mathrm{Zn}+{ }^{70} \mathrm{Zn}$ (black circles) $\mathrm{QP}$ and $\mathrm{QT} \mathrm{N} / \mathrm{Z}$ values compared to the projectile/ target/composite system $\mathrm{N} / \mathrm{Z}$ value (black line). Middle row: ${ }^{70} \mathrm{Zn}+{ }^{64} \mathrm{Zn}$ (red triangles) and ${ }^{64} \mathrm{Zn}+{ }^{70} \mathrm{Zn}$ (green inverted triangles) $\mathrm{QP}$ and $\mathrm{QT} \mathrm{N} / \mathrm{Z}$ values compared to the ${ }^{70} \mathrm{Zn}$ (black line), ${ }^{64} \mathrm{Zn}$ (dark blue line) and composite system (light green thick line) $\mathrm{N} / \mathrm{Z}$ values. Bottom row: ${ }^{64} \mathrm{Zn}+{ }^{64} \mathrm{Ni}$ (pink stars) and ${ }^{64} \mathrm{Ni}+{ }^{64} \mathrm{Zn}$ (light blue diamonds) $\mathrm{QP}$ and $\mathrm{QT} \mathrm{N} / \mathrm{Z}$ values compared to the ${ }^{64} \mathrm{Ni}$ (brown line), ${ }^{64} \mathrm{Zn}$ (blue line) and composite system (green thick line) $\mathrm{N} / \mathrm{Z}$ values.

Recent calculations $[24,25]$ show that equilibration as a function of impact parameter is achieved faster with an "asy-soft" nEOS than an "asystiff" nEOS. For example figure 2 shows the asymmetry (N/Z) of the quasiprojectile (QP) and the quasitarget (QT) from a series of iBUU calculations using either an "asy-soft" or an "asy-stiff" EOS. The top panels are 
for the symmetric ${ }^{70} \mathrm{Zn}+{ }^{70} \mathrm{Zn}$ reaction, so the results for the $\mathrm{QP}$ and $\mathrm{QT}$ are nearly identical. The middle panel shows the equilibration that takes place in the charge symmetric ${ }^{70} \mathrm{Zn}+{ }^{64} \mathrm{Zn}$ (and ${ }^{64} \mathrm{Zn}+{ }^{70} \mathrm{Zn}$ ) systems. The bottom row represents the mass symmetric systems of ${ }^{64} \mathrm{Zn}+{ }^{64} \mathrm{Ni}$ and ${ }^{64} \mathrm{Ni}+{ }^{64} \mathrm{Zn}$. As the impact parameter changes from a very peripheral interaction, with small contact time, to mid impact parameter collisions, representing reactions with increasing contact time between the QP and QT, the nucleon exchange results in the difference in the asymmetry of the reacting partners decreasing. This can been seen for both the asy-soft and the asy-stiff calculations, but the equilibration is much faster for the asy-soft interactions. The asy-stiff interaction also results in a more neutron rich QT and QP. The asy-soft interaction reaches equilibration at a value of $\mathrm{N} / \mathrm{Z}$ that is just slightly lower than that of the composite system, while the asy-stiff interaction approaches an equilibration point that is between the composite system and the $\mathrm{N} / \mathrm{Z}$ of the more neutron-rich reaction partner. Additionally it has been seen that equilibration is faster for the lower beam energies and for momentum independent interaction. [25]

Various measurements [25-30] have demonstrated migration of neutrons to the neck region and an enhancement of the asymmetry of light clusters at mid rapidity due to nucleon exchange. In general when these measurements have been compared to models they tend to agree better with asy-stiff parametrizations of the nEOS. While there were various measurements of asymmetry of the charged particles that came from the QP, and reconstruction of the QP by measurement of all the charged particles coming from the QP, Kohley made a direct measure of the QP using the MoNA-Lisa-Sweeper setup. In comparison with CoMD calculations he was able to say the data agreed best with an $\mathrm{L}=78$. [31] In a study of the $\mathrm{N} / \mathrm{Z}$ effects in competition between binary reactions and complete fusion a higher probablility for fusion was found using neutron-rich systems. This same data in comparison with CoMD agrees better with a "stiff" symmetry energy. [32]

Equilibration of the $\mathrm{N} / \mathrm{Z}$ asymmetry can also be measured by the breakup of a deformed quasiprojectile as done by Brown. [33] The average asymmetry as a function of the rotation angle allows one to measure the equilibration as a function of time. There is a clear decrease in the asymmetry of the light fragment as a function of time. [33,34] Recent analysis was performed on data from the ${ }^{70} \mathrm{Zn}+{ }^{70} \mathrm{Zn}$ reaction at $35 \mathrm{MeV} /$ nucleon using the NIMROD array. Events were selected where at least two mass identified fragments were detected. The events were sorted based on proton number followed by atomic number, with the heaviest fragment designated as $\mathrm{Z}_{H}$ and the second heaviest as $\mathrm{Z}_{L}$. In order to ensure the fragments cor- 


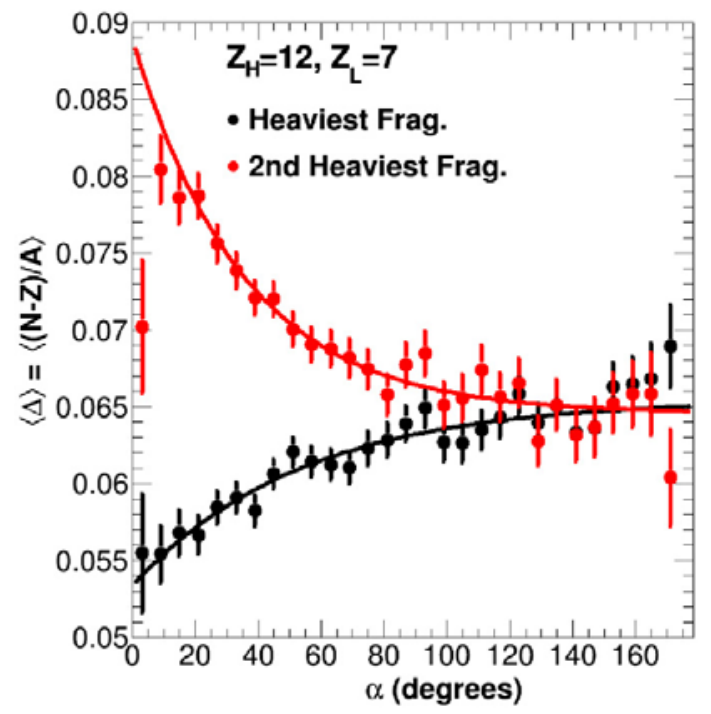

Figure 3: The (N-Z)/A of the heaviest fragment and the second heaviest fragment from an excited QP as a function of the degree of rotation of the system, a measure of its equilibration time.

responded to a large fraction of the initial projectile, events were required to have $\mathrm{Z} \geq 7$ for $\mathrm{Z}_{H}$ and $\mathrm{Z} \geq 3$ for $\mathrm{Z}_{L}$. A total $\mathrm{Z}$ cut of minimum $\mathrm{Z}=21$ (70\% of beam) and maximum $\mathrm{Z}=32$ was also included. The angular distributions for pairing of $\mathrm{Z}_{H}=12$ and $\mathrm{Z}_{L}=7$ are presented in figure 3. A large difference between the $\mathrm{N} / \mathrm{Z}$ of the $\mathrm{Z}_{H}$ and the asymmetry of the $\mathrm{Z}_{L}$ at low rotation angle is consistent with previous findings, indicating a timescale of dynamical binary splitting of the excited projectile-like fragment much shorter than its rotational period. As the rotation angle increases there is clear equilibration of the asymmetry between the heavy fragment and the light fragment. This data is currently being analyzed to extract the rate constant for equilibration. Since calculations have demonstrated that the equilibration time is sensitive to the symmetry energy of the nEOS and now we have a mechanism to sensitively measure the equilibration as a function of time future work on isospin equilibration may help to further constrain the symmetry energy of the nEOS. 


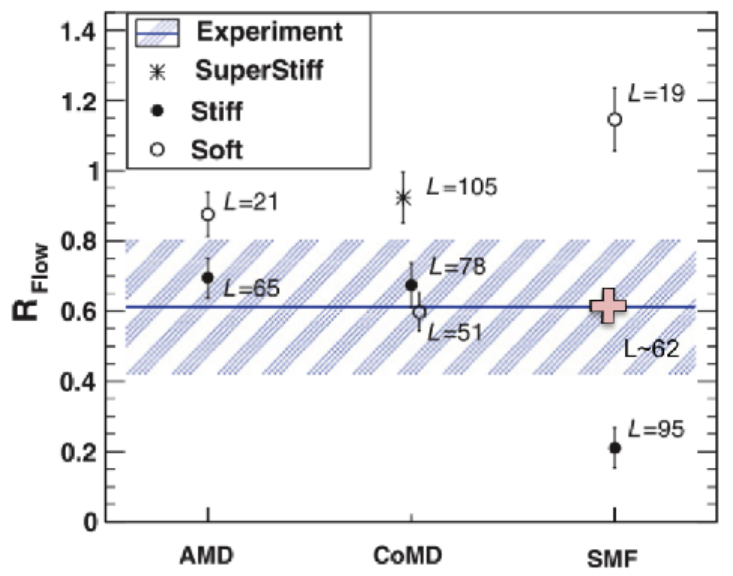

Figure 4: $\mathrm{R}_{\text {flow }}$ value from mid-peripheral reactions for various symmetry energy parameterizations of the AMD, CoMD, and SMF models. The corresponding symmetry energy slope $(\mathrm{L})$ is shown next to each calculation. The experimental value is represented by the solid blue line with the statistical uncertainty shown as the hatched blue area.

\section{Intermediate mass fragment flow}

Another method of assessing the symmetry energy in the nEOS is measurement of the transverse collective flow of light charged particles and intermediate mass fragments. Work by Kohley compared the transverse collective flow of IMFs for mid-peripheral reactions in the $35 \mathrm{MeV} /$ nucleon ${ }^{70} \mathrm{Zn}+{ }^{70} \mathrm{Zn}$, ${ }^{64} \mathrm{Zn}+{ }^{64} \mathrm{Zn}$, and ${ }^{64} \mathrm{Ni}+{ }^{64} \mathrm{Ni}$ systems to AMD, CoMD and SMF model predictions with various parameterizations of the nEOS. Using the mid impact parameter events - when the flow signatures are largest - a comparison was performed to AMD, CoMD and SMF as shown in figure 4. For all calculations agreement was better with an asy-stiff parametrization rather than an asy-soft one. All calculations agree with an L of about 60. [35] This same data showed a better agreement with the AMD asy-stiff parametrization when compared over a variety of impact parameters. [36]

\section{$5 \quad$ Future directions}

There have been many proposed observables and various experiments and theoretical simulations used to explore the symmetry energy in the nEOS. We need to understand the differences in the various model predictions 
and continue to explore observables or combinations of observables with increased power to discriminate between various theoretical predictions. Higher order correlation of variables can provide more robust signatures. [37] Additionally, higher energy collisions can provide constraints at higher densities. [38-40] While this has all been done in the realm of neutron and protons as the relevant elemental particles, recent theoretical work by DiToro [41] suggests that for very isospin asymmetric matter we can expect a much earlier transition to the quark phase. This may help to explain the recent neutron star data that requires a softening of the $\mathrm{nEOS}$ for the core in order to explain the measurements of neutron star radii.

\section{Acknowledgements}

This work was supported in part by the US Department of Energy under grant DE-FG02-93ER40773 and the Robert A. Welch Foundation under grant A-1266.

\section{References}

[1] J.M. Lattimer, M. Prakash, Science 304, 536 (2004)

[2] J.M. Lattimer, Ann. Rev. Nucl. Part. Sci. 62, 485 (2012)

[3] P. Demorest et al., Nature 467, 1081 (2010)

[4] D.H. Youngblood et al., Phys. Rev. Lett. 82, 691 (1999)

[5] E. Khan et al., Phys. Rev. Lett. 109, 092502 (2012)

[6] P. Danielewicz, R. Lacey, W.G. Lynch, Science 298, 1592 (2002)

[7] S.J.Y. D. V. Shetty, G.A. Souliotis, Phys. Rev. C 75, 034602 (2007)

[8] M.B. Tsang et al., Phys. Rev. Lett. 92, 062701 (2004)

[9] A. Ono et al., Phys. Rev. C 68, 051601(R) (2003)

[10] D.V. Shetty et al., Phys. Rev. C 70, 011601(R) (2004)

[11] D.V. Shetty, S.J. Yennello, G.A. Souliotis, Phys. Rev. C 76, 024606 (2007)

[12] A.B. McIntosh et al., Phys. Lett. B 719, 337 (2013) 
[13] A.B. McIntosh et al., Phys. Rev. C 87, 034617 (2013)

[14] A.B. McIntosh et al., EPJA 50, 35 (2014)

[15] A.B. McIntosh et al., Progress in Constraining the Asymmetry Dependence of the Nuclear Caloric Curve, in Proceedings of the NN2015 Conference (2015)

[16] S. Wuenschel et al., Phys. Rev. C 79, 061602(R) (2009)

[17] S. Galanopoulos et al., Nucl. Phys. A 837, 145 (2010)

[18] R. Tripathi et al., Phys. Rev. C 83, 054609) (2011)

[19] J. Mabiala et al., Int. J. Mod. Phys. E 22, 1350090 (2013)

[20] S. Yennello et al., Phys. Lett B. 15, 321 (1994)

[21] H. Johnston et al., Phys. Lett. B 371, 187 (1996)

[22] F. Rami et al., Phys. Rev. Lett. 84, 1116 (2000)

[23] L. Shi, P. Danielewicz, Phys. Rev. C 68, 064604 (2003)

[24] L. May, Ph.D. thesis, Texas A\&M Univ. (2015)

[25] Rizzo et al., Nucl. Phys. A 806, 79 (2008)

[26] D. Theriault et al., Phys. Rev. C 74, 051602(R) (2006)

[27] E.D. Filippo et al., Acta Physica Polonica B 37, 199 (2006)

[28] P. Russotto et al., Procs of IWM 05 105, 13 (2006)

[29] E.D. Filippo et al., Phys. Rev. C 86, 014610 (2012)

[30] Galichet et al., Phys. Rev. C (2010)

[31] Z. Kohley et al., Phys. Rev. C 88, 041601 (2013)

[32] G. Cardella et al., Phys. Rev. C 85, 084609 (2012)

[33] K. Brown et al., Phys. Rev. C 87, 061601 (2013)

[34] K. Stiefel et al., Phys. Rev. C 90, 061605 (2014)

[35] Z. Kohley et al., Phys. Rev. C 85, 064605 (2012) 
[36] Z. Kohley et al., Phys. Rev. C 86, 044605 (2012)

[37] P. Cammarata et al., Nucl. Instr. Meth. A 761, 1 (2014)

[38] P. Russotto et al., JoP conference series 420, 012092 (2013)

[39] G. Jhang et al., ARIS conference proceedings (2014)

[40] R. Shane et al., Nucl. Instr. Meth. A 784, 512 (2015)

[41] W. DiToro et al., Phys. Rev. C 83, 014911 (2011) 